\title{
Benchmarking of DFT methods using experimental free energies and volumes of activation for the cycloaddition of alkynes to cuboidal $\mathrm{Mo}_{3} \mathrm{~S}_{4}$ clusters
}

\author{
Elena Pedrajas, ${ }^{1,2}$ José A. Pino-Chamorro, ${ }^{1}$ Montserrat Ferrer, ${ }^{3}$ M. Jesús Fernández-Trujillo, ${ }^{1}$ Rosa \\ Llusar, ${ }^{2}$ Manuel Martínez, ${ }^{3}$ Manuel G. Basallote, ${ }^{1}$ Andrés G. Algarra ${ }^{1}$ \\ Correspondence to: Andrés G. Algarra (E-mail: andres.algarra@uca.es)
}

\begin{abstract}
${ }^{1}$ Elena Pedrajas, José A. Pino-Chamorro, M. Jesús Fernández-Trujillo, Manuel G. Basallote, Andrés G. Algarra Departamento de Ciencia de los Materiales e Ingeniería Metalúrgica y Química Inorgánica, Universidad de Cádiz, Apartado 40, Puerto Real, 11510 Cádiz, Spain

${ }^{2}$ Elena Pedrajas, Rosa Llusar Departament de Química Física i Analítica, Universitat Jaume I, Av. Sos Baynat s/n, 12071 Castelló, Spain

${ }^{3}$ Montserrat Ferrer, Manuel Martínez

Departament de Química Inorgànica i Orgànica, Secció de Química Inorgànica, Universitat de Barcelona, Martí i Franquès 1-11, E-08028 Barcelona, Spain
\end{abstract}

\begin{abstract}
Herein, the kinetics of the concerted [3+2] cycloaddition reaction between the $\left[\mathrm{Mo}_{3}\left(\mu_{3}-S\right)(\mu-\right.$ $\left.\mathrm{S}_{3} \mathrm{Cl}_{3}(\mathrm{dmen})_{3}\right]^{+}$(dmen=N, $\mathrm{N}^{\prime}$-dimethyl-ethylenediamine) $\left([\mathbf{1}]^{+}\right)$cluster and various alkynes to form dithiolene derivatives is thoroughly studied, with measurements at different temperatures and pressures allowing the determination of the free energies and volumes of activation. These parameters, together with the available single crystal X-ray diffraction structures are employed to test a number of commonly used DFT methods from across the Jacob's ladder, as well as the effects associated with the size of the basis sets, the way in which solvent effects are taken into account, or the inclusion of dispersion effects. All in all, a protocol that leads to average deviations between experimental and computed $\Delta V^{\#}$ and $\Delta G^{\#}$ values similar to the uncertainty of the experimental measurements is obtained.
\end{abstract}

\section{Introduction}

Theory is expected to provide answers to the many questions related with the mechanism of chemical reactions. This goal requires of accurate computational methods, and for that purpose an adequate estimation of the activation parameters is critical. In this sense, recent analyses have highlighted that obtaining reliable and predictive mechanistic insight from modelling remains challenging. ${ }^{1-2}$ As the kinetic and mechanistic features of reactions are best evaluated by using activation parameters, an adequate refinement of computational methods for evaluating them is highly desired.
Given the difficulties of the current methodology to separate enthalpic and entropic contributions, estimation of the experimental activation free energies is surely the best way to test the performance of computational methods. The activation volume, another activation parameter typically less used because of its higher experimental demands, is also fundamental for obtaining an adequate mechanistic picture of reactions, and so the development of adequate procedures for obtaining accurate computational estimations is also pursued. In the present work we tested the capability of the most commonly used DFT methods to model the activation parameters of 
a series of concerted reactions between $\mathrm{Mo} / \mathrm{S}$ species and alkynes.

Mo ${ }^{\text {IV }}$ combines with sulfur to generate robust clusters with a $\left[\mathrm{Mo}_{3}\left(\mu_{3}-\mathrm{S}\right)(\mu-\mathrm{S})_{3}\right]^{4+}$ core..$^{3-4}$ These structures thus feature two types of sulfur ligands, with each metal centre being coordinated to the capping $\left(\mu_{3}-S\right)$ and two bridging $(\mu-S)$ ligands (see Scheme 1). Moreover, each metal centre features a distorted octahedral geometry if the Mo-Mo bonds are ignored, and therefore three additional coordination sites are normally occupied by mono- and/or polydentate ligands. Besides the reactivity associated to the Mo(IV) centres and its outer coordination environment, ${ }^{5}$ the three bridging $(\mu-S)$ ligands provide important reactivity paths. In fact, two main processes are known to occur at these sulphur sites: a) incorporation of a second metal $\left(\mathrm{M}^{\prime}\right)$ to form heterometallic $\left[\mathrm{Mo}_{3} \mathrm{M}^{\prime} \mathrm{S}_{4}\right]^{4+}$ clusters $^{6}$; b) alkyne insertion to produce dithiolene adducts, as shown in Scheme $1 .^{7}$ According to previous findings, such insertion takes place through a concerted [3+2] cycloaddition process between a $\left[\mathrm{Mo}(\mu-\mathrm{S})_{2}\right]$ cluster moiety and the two $s p$ carbon atoms of the alkyne, as illustrated in Scheme $1 .^{7}$

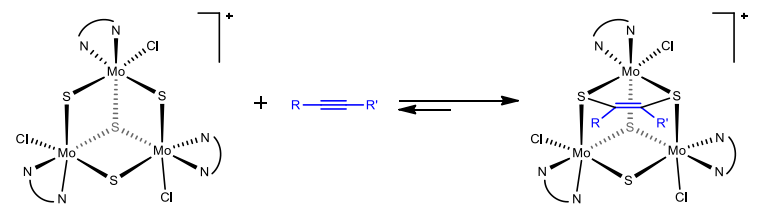

Scheme 1. [3+2] cycloaddition reaction between $[1]^{+}$and alkynes (dmen ligands represented as $\left.\mathrm{N}^{\wedge} \mathrm{N}\right)$.

From a computational point of view, although DFT results have been used to explain all the observed trends in reactivity, we have found inconsistencies associated with errors of up to 5-6 $\mathrm{kcal} / \mathrm{mol}$ in the predicted free energies. In this manuscript we have taken advantage of the clean reaction between the $[\mathbf{1}]^{+}$cluster and some alkynes, dimethylacetylene dicarboxylate (dmad) and phenylacetylene (PhA), to identify which of the commonly used computational approaches produce the most accurate results. To do so, not only have we used the interatomic distances calculated from their single crystal Xray diffraction structures, but also free energy barriers and activation volumes kinetically determined. In this way, an optimised computational strategy has been developed that yields estimations of activation parameters that are typically within the standard deviation of the experimental determinations. Furthermore, the protocol was tested on other $[3+2]$ cycloaddition reactions of $\left[\mathrm{Mo}_{3} \mathrm{~S}_{4}\right]^{4+}$ cuboidal clusters with similar results, thus highlighting its versatility. Thus, the conclusions of this work constitute an excellent starting point towards future calculations on catalytic processes involving these clusters.

Additionally, it is worth noting that such clusters are important in relation to Group-VI transition metal dichalcogenides (TMDs), a class of materials thoroughly studied nowadays due to their ability to catalyse the Hydrogen evolution reaction (HER). ${ }^{8}$ The location of the catalytic active sites of these materials is however still under debate, and in this sense, the use of homogeneous analogues able to mimic their structure has been cleverly used by some researchers to shed light into this dilemma. ${ }^{9}$ Among those model species, $\left[\mathrm{Mo}_{3}\left(\mu_{3}-\mathrm{S}\right)(\mu-\mathrm{S})_{3}\right]^{4+}$ clusters share structural similarities with the basal planes of $\mathrm{MoS}_{2}{ }^{10}$ and therefore any improvement in their computational characterisation will reverberate in a better understanding of HER catalysts.

\section{Methods}

\section{Chemical substances}

The cluster $\left[\mathrm{Mo}_{3} \mathrm{~S}_{4} \mathrm{Cl}_{3}(\mathrm{dmen})_{3}\right]\left(\mathrm{BF}_{4}\right), \quad[1]\left(\mathrm{BF}_{4}\right)$, was prepared according to literature procedures. ${ }^{11-12}$ All other chemicals were reagent grade commercially available and were used as received. 


\section{Kinetic experiments}

Ambient pressure kinetic experiments were carried out using an Applied Photophysics SX18MV stopped-flow spectrophotometer provided with a PDA1 photodiode array detector, and with a Cary 50 Bio UV-Vis spectrophotometer for conventional measures. All experiments were carried out at the desired temperatures in acetonitrile solution by mixing a stock solution of the cluster $\left(2-5 \times 10^{-4} \mathrm{M}\right)$ with another solution containing the alkyne in a concentration range large enough (0.01-0.050 $M)$ to ensure pseudo-first order conditions of alkyne excess. Some experiments at two different cluster concentrations were also carried out to confirm the first order dependence of the observed rate constants on its concentration. Runs at variable high pressure were conducted with the same procedures, but using the pressurising systems and cells described previously. ${ }^{13}$

All time-resolved data were collected as full (300-1000 nm) spectra and treated with the standard Specfit or Reactlab Kinetics software. ${ }^{14-15}$ Observed rate constants were obtained from the full time-resolved spectral changes or alternatively at the wavelength were a maximum change was observed (ca. 400 or $900 \mathrm{~nm}$ ). For the vast majority of the runs the changes agreed with the operation of an $A \rightarrow B$ single exponential equation when pseudo-first order conditions applied, and were fitted accordingly. In cases where a drift of the absorbance values was observed, the data were fitted to two consecutive exponentials but the values derived for the second rate constants showed erratic changes and were disregarded.

\section{Computational details}

All calculations were performed with Gaussian 09, Revision D.01. ${ }^{16}$ Optimisations were carried out in the gas phase without any symmetry constraint, and employed an integration grid obtained with 99 radial shells and 590 angular points per shell centred on each atom, which is denoted as "ultrafine" in Gaussian 09. Vibrational frequencies were computed using
Table 1. Summary of basis set systems used in this work.

\begin{tabular}{|c|c|c|c|}
\hline $\begin{array}{c}\text { Basis } \\
\text { set } \\
\text { system }\end{array}$ & Mo centres & $S$ centres & $\begin{array}{c}\mathrm{Cl}, \mathrm{N}, \mathrm{O}, \mathrm{C} \text { and } \\
\mathrm{H} \text { centres }\end{array}$ \\
\hline BS1 & $\mathrm{SDD} / \mathrm{ECP}^{[\mathrm{a}]}$ & $\begin{array}{l}\mathrm{SDD} / \mathrm{ECP}^{[\mathrm{a}]} \\
+\mathrm{Pol}^{[\mathrm{b}]} \\
\mathrm{SDD} / \mathrm{ECP}^{[\mathrm{a}]}\end{array}$ & $6-31 G(d, p)$ \\
\hline BS2 & $\mathrm{SDD} / \mathrm{ECP}^{[\mathrm{a}]}$ & $+\mathrm{Pol}^{[\mathrm{b}]}$ & $6-311+G(2 d, 2 p)$ \\
\hline BS3 & $\begin{array}{l}\text { SDD/ECP } \\
\text { cc-pVDZ- }\end{array}$ & D95 & \\
\hline BS4 & $\mathrm{PP}^{[\mathrm{c}]}$ & $c c-p V T Z^{[d]}$ & $c c-p V D z^{[e]}$ \\
\hline BS5 & Def2-TZVP ${ }^{[t]}$ & Def2-TZVP ${ }^{[t]}$ & Def2-TZVP ${ }^{[t]}$ \\
\hline \multicolumn{4}{|c|}{$\begin{array}{l}\text { [a] SDD/ECP = Stuttgart / Dresden ECP, see Ref. 19. [b] } \\
\text { Added polarisation function ( } \zeta=0.503 \text { ), see Ref. 20. [c] Ref. } \\
\text { 24. [d] Ref. 25. [e] Ref. 26. [f] Ref. } 23 .\end{array}$} \\
\hline
\end{tabular}

the harmonic oscillator approximation on all optimised geometries at $1 \mathrm{~atm}$ and $298.15 \mathrm{~K}$. This served to characterise them as either minima or transition states, as well as to obtain the thermal and entropic corrections required to further calculate free energy differences. The connection of transition states with the corresponding reactants and products was confirmed via intrinsic reaction coordinate (IRC) calculations $^{17-18}$ and subsequent optimisation to minima. Unless otherwise stated, BS1 was employed for optimisation purposes and BS2 to obtain improved energetic values via singlepoint calculations. A summary of how each basis set system is constructed is included in Table 1. BS1 uses the Stuttgart RECPs and corresponding basis sets to describe the Mo and $S$ centres, ${ }^{19}$ with added polarisation on the latter $(\zeta=0.503),{ }^{20}$ whereas the Pople style 6$31 \mathrm{G}(\mathrm{d}, \mathrm{p})$ basis set is used for all other atoms $(\mathrm{Cl}$, $\mathrm{N}, \mathrm{O}, \mathrm{C}, \mathrm{H}$ ). Mo and $\mathrm{S}$ centres are described similarly in BS2, which only differs from BS1 in that the remaining atoms are modelled using the $6-311+G(2 d, 2 p)$ basis set. Nevertheless, given that previous DFT studies on reactions involving Mo centres have shown a significant dependence of results on the quality of the basis set, ${ }^{21-22}$ additional optimisations and/or single-point calculations were performed with the basis set systems indicated in Table 1 . This includes common basis set systems such as BS3, invoked in Gaussian software by using the SDD command, a combination of Dunning's correlation consistent basis sets and effective 
core potentials in $\mathrm{BS} 4$, or the triple- $\zeta$ valence basis set including polarisation functions Def2TZVP optimised by Weigend and Ahlrichs. ${ }^{23}$

A number of popular density functionals across the Jacob's ladder ${ }^{27}$ were employed and they are included in Table 2. On each case, both optimisations and subsequent single-point calculations were carried out with the same density functional. The latter single-point calculations at the DFT/BS2 level included the effects of the solvent (acetonitrile) using either the IEFPCM ${ }^{28}$ (abbreviated PCM) or the SMD $^{29}$ approaches.

Dispersion effects were taken into account in different ways. No additional correction was applied to those functionals that already include these in some way, such as B97D3, WB97XD. For the remaining functionals, both Grimme's D3(0) and D3(BJ) dispersion corrections were computed as single-point corrections whenever possible. Note that for the M06 series only the D3(0) correction could be applied due to the lack of Becke-Johnson parameters. In addition, in some cases Grimme's D3(0) and D3(BJ) corrections were included self-consistently in the optimisation procedure, leading to the $B P 86 D 3(B J)$, B3LYPD3(BJ), PBEOD3(0) and PBEOD3(BJ) combinations. Finally, no dispersion corrections were computed for the M11 functional.

A number of additional corrections were also considered in order to calculate activation free energies. Given that all reactions investigated take place in solution, a standard state of $1 \mathrm{M}$ was employed. For that purpose, the quantity of $R \ln (24.46), 6.354 \mathrm{cal} / \mathrm{mol} \cdot \mathrm{K}$, was subtracted from the raw entropies obtained from Gaussian 09 for each computed species. ${ }^{30}$ Note that, in the present case, this subtraction results in a correction term of $1.89 \mathrm{kcal} / \mathrm{mol}$ for each species, which in turn leads to the same 1.89 $\mathrm{kcal} / \mathrm{mol}$ energy decrease on the free energy barrier $\left(\Delta G^{\#}\right)$ for the forward reaction between cluster and alkynes, with the correction cancelling out in the case of the reverse barrier. On the other hand, the molecular symmetry of the species has an impact on their rotational entropy. All optimised species in this study were

\begin{tabular}{|c|c|c|c|}
\hline Name & Type $^{[a]}$ & $X^{[b]}$ & Ref. \\
\hline BP86 & GGA & 0 & $33-34$ \\
\hline BP86D3(BJ) ${ }^{[c]}$ & $\mathrm{GGA}+\mathrm{D}$ & 0 & $33-36$ \\
\hline B97D3 ${ }^{[c]}$ & $G G A+D$ & 0 & 37 \\
\hline TPSS & mGGA & 0 & 38 \\
\hline M06-L & mGGA & 0 & 39 \\
\hline TPSSh & GH-mGGA & 10 & $40-41$ \\
\hline B3LYP & GH-GGA & 20 & 42 \\
\hline B3LYPD3(BJ) ${ }^{[c]}$ & GH-GGA+D & 20 & 42 \\
\hline PBE0 & GH-GGA & 25 & $43-44$ \\
\hline PBE0D3(0) ${ }^{[c]}$ & GH-GGA+D & 25 & $\begin{array}{l}35-36 \\
43-44\end{array}$ \\
\hline PBE0D3(BJ) ${ }^{\lfloor c\rfloor}$ & $\mathrm{GH}-\mathrm{GGA}+\mathrm{D}$ & 25 & $\begin{array}{l}35-36 \\
43-44\end{array}$ \\
\hline M06 & GH-mGGA & 27 & 45 \\
\hline M06-2X & GH-mGGA & 54 & 45 \\
\hline M06-HF & GH-mGGA & 100 & 46 \\
\hline M11 & $\mathrm{RSH}-\mathrm{mGGA}$ & $42.8-100$ & 47 \\
\hline wB97XD ${ }^{[d]}$ & $\begin{array}{l}\mathrm{RSH}- \\
\mathrm{GGA}+\mathrm{D}\end{array}$ & $22.2-100$ & $48-49$ \\
\hline \multicolumn{4}{|c|}{$\begin{array}{l}\text { [a] GGA = generalised-gradient approximation; +D = addition } \\
\text { of molecular mechanic dispersion corrections; GH-GGA = } \\
\text { global hybrid GGA, GGA plus some percentage of nonlocal } \\
\text { HF exchange; mGGA = meta-GGA, GGA plus local kinetic } \\
\text { energy density and some percentage of nonlocal HF } \\
\text { exchange; RSH = range-separated hybrid. [b] } X \text { denotes de } \\
\text { percentage of Hartree-Fock exchange. A single value } \\
\text { indicates a local }(X=0) \text { or hybrid }(X \neq 0) \text { functional, whereas } \\
\text { two values indicate } X \text { at short and long inter-electronic } \\
\text { separations. [c] These functionals include Grimme's D3(BJ) } \\
\text { correction, see Ref. } 35-36 \text {. [d] This functional uses a version } \\
\text { of Grimme's D2 dispersion correction, see Ref. } 37 \text {. }\end{array}$} \\
\hline
\end{tabular}

computed with C1 symmetry, and therefore the effect of symmetry was subsequently added to the free energy. This was carried out by including the term $R \ln (\sigma)$, where $\sigma$ represents the symmetry number. ${ }^{31-32}$ In particular, symmetry numbers of 3 and 2 were used for cluster and alkynes, respectively, which led to contributions of 0.65 and $0.41 \mathrm{kcal} / \mathrm{mol}$. A symmetry number of 1 was used for the 
transition states and dithiolene products, thus resulting in no additional correction for these species. As a result, this symmetry correction leads to a decrease of $1.06 \mathrm{kcal} / \mathrm{mol}$ on the free energy barrier for the forward reaction between cluster and alkyne.

All in all, the activation free energies reported in the text at a DFT2/BSX(solvent)//DFT1/BSY(gas) $(X, Y=1-5$; solvent= $P C M, S M D)$ level of theory are based on the electronic energies computed at the DFT2/BSX(solvent) level (note that in those cases in which DFT2 corresponds to a dispersion corrected functional, such dispersion correction is included in this term), and also include the free energy correction computed at the DFT1/BSY(gas) level, the standard state correction to change from $1 \mathrm{~atm}$ to $1 \mathrm{M}$, and the correction to account for the symmetry of the molecules. The molecular vdW volumes required to compute the $\Delta V^{\dagger}$ values were obtained using the default Gaussian09 parameters for PCM and SMD solvation models, i.e. scaled $(f=1.1)$ UFF and unscaled intrinsic atomic Coulomb radii, respectively.

A summary of the computed energies and vdW volumes for all optimized species is included in the Supporting Information, whereas their structures are available at the ioChem BD database, ${ }^{50}$ where they can be accessed via https://doi.org/10.19061/iochem-bd-6-34.

\section{Results and discussion}

\section{Kinetics of the reaction of $[1]^{+}$with alkynes}

The reaction of $[\mathbf{1}]^{+}$with an excess of various alkynes (adc, dmad, PhA, DPhA, PrA) was preliminary investigated (adc = acetylenedicarboxylic acid; PrA = propargyl alcohol; $\mathrm{DPhA}=$ diphenylacetylene; $\operatorname{Pr} \mathrm{A}=$ propargyl alcohol). While no reaction with DPhA was observed even after 4 days at $60 \stackrel{\circ}{ } \mathrm{C}$, the reaction with PhA and PrA occurred in a few hours at room temperature, and in seconds when adc and dmad were used (the kinetics of the reaction with dmad at $250 \mathrm{C}$ has been previously reported). ${ }^{10}$ The typical spectral changes observed for the reaction of $[\mathbf{1}]^{+}$with

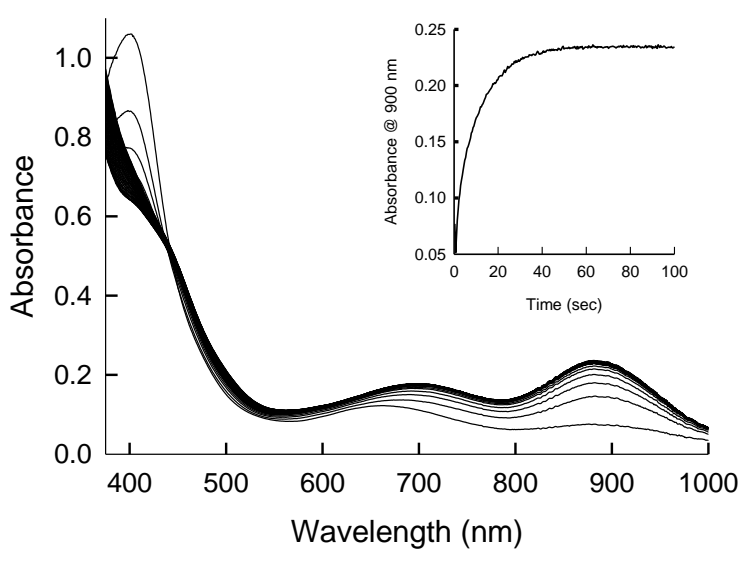

Figure 1. Typical spectral changes for the reaction of cluster [1] $]^{+}$with dmad in acetonitrile solution $\left(25.0\right.$ o $\mathrm{C},[1]^{+}=4.57 \times 10-4 \mathrm{M}$, [dmad] = $0.016 \mathrm{M})$.
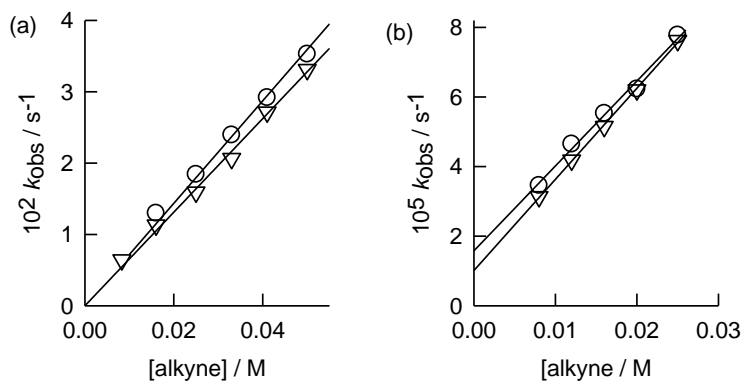

Figure 2. Representative plots of the dependence of the observed rate constants for the reaction of $[\mathbf{1}]^{+}$with different alkynes in acetonitrile solution at 25.0 ㅇ (a): dmad (circles) and adc (triangles); b): PhA (circles) and PrA (triangles).

an excess of dmad in acetonitrile solution are shown in Figure 1. A significant change in the intensity of the bands at 400 and $660 \mathrm{~nm}$ is evident, as well as the appearance of a new band at $885 \mathrm{~nm}$, which is a characteristic feature of the dithiolene addition products. ${ }^{7}$

Similar spectral changes are observed with the other alkynes, and in all cases they can be fitted to a single exponential $A \rightarrow B$ model, i.e. no reaction intermediate build-ups in significant concentration during the cycloaddition process. The concentration dependence of the obtained 
pseudo-first order rate constants ( $k_{\mathrm{obs}}$, collected in Table S1 of the supporting information) are shown in Figure 2 for the different systems studied. Interestingly, the data show a non-zero intercept for the PhA and PrA alkyne systems, i.e. those showing the reactivity in the hours time scale, whereas the faster reacting activated dmad and adc alkynes feature a zero intercept. Such dependences can be explained by the rate law in eq 1 , where $k_{+}$and $k_{-}$ correspond to the forward and reverse rate constants for the formation of the cycloaddition products in an equilibrium process. The equilibrium nature of the reactions with PhA and PrA is confirmed by the increasing magnitude of the absorbance changes when the alkyne concentration is increased. The reactions with dmad and adc can be considered to represent irreversible processes under the experimental conditions used. Table 3 collects the relevant kinetic parameters for all the systems studied $\left(k_{-}\right.$and $\left.k_{+}\right)$as well as the calculated equilibrium constants for the process ( ${ }^{\text {calc }} K_{\text {eq }}=k_{+} / k_{-}$).

$k_{\mathrm{obs}}=k_{-}+k_{+} \cdot[$ alkyne $]$

Table 3. Summary of kinetic and equilibrium parameters obtained for the reaction of $[1]^{+}$with the different alkynes studied. ${ }^{[a]}$

\begin{tabular}{llllc} 
Parameter & \multicolumn{1}{c}{ dmad } & \multicolumn{1}{c}{ PhA } & \multicolumn{1}{c}{ PrA } & \multicolumn{1}{c}{ adc } \\
${ }^{298} \mathrm{~K}_{+} / \mathrm{M}^{-1} \mathrm{~s}^{-1}$ & $0.72(2)$ & $1.53(5) \cdot 10^{-3}$ & $2.62(4) \cdot 10^{-3}$ & $0.65(1)$ \\
${ }^{298} \mathrm{~K} / \mathrm{s}^{-1}$ & - & $2.6(1) \cdot 10^{-5}$ & $1.01(7) \cdot 10^{-5}$ & \\
$\log ^{\text {calc }} K_{\text {eq }}$ & - & $2.51(6)$ & $3.2(1)$ &
\end{tabular}

[a] Numbers in parenthesis are errors in the last significant digit.

The variation of the kinetic parameters with temperature and pressure were also determined for the PhA and dmad systems, as representatives of the two types of kinetic trends in Figure 2. Figure 3 shows the Eyring and $\ln k / P$ plots for the systems studied (see also Figures S1 and S2), whereas Table 4 collects the corresponding thermal and pressure activation parameters derived. The activation enthalpy for the cycloaddition (forward process) is lower in the case of dmad, and the negative activation entropies are, in both cases, in agreement with an ordering on going to the transition state, a fact that is also shown by the negative activation volumes in the non-polar, non-protic media used for the study (acetonitrile). For the reaction with PhA the data even allow for a comprehensive description of the reversible process. The activation parameters for the forward and backward reaction lead to a reasonable estimation of the corresponding thermodynamic $\Delta H_{0}=6.2 \pm 2.6 \mathrm{~kJ} \mathrm{~mol}^{-1}, \Delta S_{0}=$ $33 \pm 8 \mathrm{~J} \mathrm{~K}^{-1} \mathrm{~mol}^{-1}$, and $\Delta V_{0}=-18 \pm 2 \mathrm{~cm}^{3} \mathrm{~mol}^{-1}$. It is important to note that the values indicate that the overall reaction is thermodynamically favoured $\left(\Delta G_{0}<0\right)$, despite an unfavourable enthalpy term, due to its entropy changes.
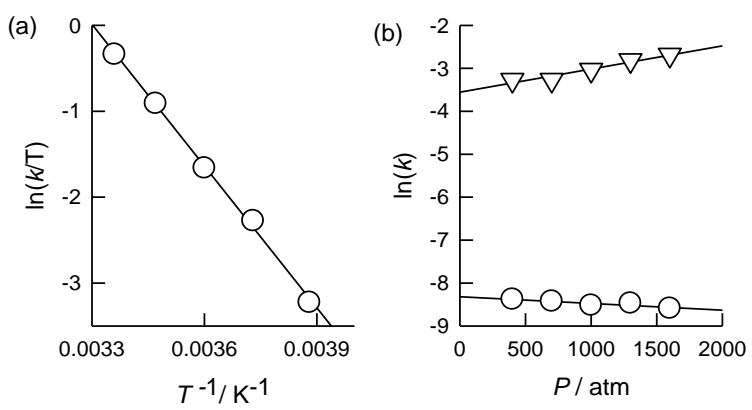

Figure 3. a) Eyring plot for the tempertaure dependence of the $k_{+}$rate constant for the reaction of the $[\mathbf{1}]^{+}$cluster with dmad; $\left.b\right) \ln (k)$ versus $\mathrm{P}$ plot for the $\mathrm{k}_{+}$(triangles) and $\mathrm{k}_{-}$(circles) rate constants for the reaction of the $[\mathbf{1}]^{+}$ cluster with PhA.

\begin{tabular}{|c|c|c|c|}
\hline Parameter & $\begin{array}{c}\text { Reaction } 1 \\
\text { PhA } \\
\text { Forward }\end{array}$ & $\begin{array}{c}\text { Reaction } 2 \\
\text { PhA } \\
\text { Reverse }\end{array}$ & $\begin{array}{c}\text { Reaction 3 } \\
\text { dmad } \\
\text { Forward }\end{array}$ \\
\hline$\Delta H^{\#} / \mathrm{kcal}$ & & & \\
\hline $\begin{array}{l}\mathrm{mol}^{-1} \\
\Delta S^{\#} / \mathrm{cal} \mathrm{K}^{-1}\end{array}$ & $19(1)$ & $12(2)$ & $9.9(1)$ \\
\hline $\begin{array}{l}\mathrm{mol}^{-1} \\
{ }^{298} \Delta G^{\#} / \mathrm{kcal}\end{array}$ & $-6(3)$ & $-39(7)$ & $-26.3(5)$ \\
\hline $\begin{array}{l}\mathrm{mol}^{-1} \\
\Delta V^{\#} / \mathrm{cm}^{3}\end{array}$ & $21(1)$ & $24(3)$ & 17.7(2) \\
\hline $\mathrm{mol}^{-1}$ & $14(2)$ & $4(1)$ & $-16(1)$ \\
\hline
\end{tabular}


[a] Numbers in parenthesis are errors in the last significant digit.

\section{Computational results on the reaction of [1] with alkynes}

The main purpose of this manuscript is to test which computational methodology is able to better model the previous experimental results. To do so, we have performed calculations aimed at determining not only the effect of the density functional, but also other important aspects such as the size of the basis set system, the inclusion of dispersion effects, or the way in which solvents effects are modelled.

\section{Optimised structures}

The X-ray diffraction structures of the cluster [1] $\left(\mathrm{BF}_{4}\right)$ and the product of its reaction with dmad ([1-dmad $]_{2}\left(\mathrm{Mo}_{6} \mathrm{Cl}_{14}\right)$ can be found in the literature. ${ }^{10-11}$ These have been employed to determine which functional leads to the most accurate optimised structures when using the basis set system BS1. The results, listed in Tables S3 and S4, show that differences between optimised and experimental interatomic distances are small, i.e. each functional performs relatively similarly for both structures. As expected, common DFT methods not including dispersion effects such as BP86 and B3LYP systematically result in expanded structures, whereas those accounting for such effects lead to more compact structures. ${ }^{51}$ This palliates the systematic overestimation of internuclear distances and reduces the general overestimation of bond lengths. The effect is clear for instance when comparing B3LYP and B3LYPD3(BJ) data. The different flavours of the Minnesota M06 family of functionals behave quite similarly, only showing a small improvement in the mean unsigned error MUE values with respect to BP86 and B3LYP.

From the data in Tables S3 and S4 it is difficult to determine a correlation between the percentage of HF exchange and the quality of the results, as dispersion effects seem to be much more important. In fact, among the two tested range-separated functionals, M11 and WB97XD, the latter gives generally better agreement with the experimental data probably because it contains a correction for dispersion

\begin{tabular}{|c|c|c|c|}
\hline $\begin{array}{l}\text { Basis } \\
\text { set } \\
\text { system }\end{array}$ & $\begin{array}{l}\text { MUE } \\
{[1]^{+}}\end{array}$ & $\begin{array}{c}\text { MUE } \\
{[\text { 1-dmad }]^{+}}\end{array}$ & $\begin{array}{l}\text { functions for } \\
{[\mathbf{1}]^{+} /[\mathbf{1}-\mathbf{d m a d}]^{+}}\end{array}$ \\
\hline BS1 & 0.010 & 0.009 & $658 / 828$ \\
\hline BS2 & 0.011 & 0.009 & $1087 / 1411$ \\
\hline BS3 & 0.034 & 0.036 & $495 / 607$ \\
\hline BS4 & 0.014 & 0.017 & $811 / 981$ \\
\hline BS5 & 0.018 & 0.018 & $1153 / 1499$ \\
\hline
\end{tabular}

effects. Interestingly, the best agreement (measured as the lowest MUE) within the functional selection herein is obtained for the PBEO and its D3(0) and D3(BJ) dispersioncorrected versions. The PBEO functional is known to give relatively accurate geometries for transition metal complexes, ${ }^{52-56}$ nevertheless, it is quite surprising that: a) it gives lower MUE values than most dispersioncorrected functionals; b) the obtained MUE values are quite insensitive to the inclusion of dispersion corrections for this functional. The consequences of including solvent effects during the optimisation procedure have also been studied at the PBEO/BS1 level via PCM and SMD approaches (see the footnotes at Tables S3 and S4). Both for $[\mathbf{1}]^{+}$and [1-dmad $]^{+}$this showed roughly no differences in terms of MUE values when compared with the gas phase optimisations. On the contrary, the accuracy of the optimised structures strongly depends on the basis sets employed. The results of these calculations, carried out in the gas phase in combination with the PBEO functional are included in Table 5 . These show that the best agreement is obtained when BS1 and BS2 are employed, with only small differences between their MUE values. Given that BS1 and BS2 only differ in the way in which $\mathrm{Cl}, \mathrm{N}, \mathrm{O}, \mathrm{C}, \mathrm{H}$ atoms are modelled, with Pople style double- and triple- $Z$ basis sets, respectively, it seems that the use of triple- $Z$ basis set in the latter is not necessary to obtain accurate geometries. On 
the contrary, the accuracy decreases significantly when BS3 is employed, thus suggesting that the Dunning/Huzinaga full double zeta basis set is not appropriate for these optimisations. BS4 was selected on the basis of a recent computational assessment of DFT methods for Mo/W-mediated reactions. ${ }^{31}$ As shown in Table 5, it leads to slightly larger MUE values than BS1, and a deeper analysis of the computed structures show that this is mostly originated by Mo-Mo and Mo-S distances ca. 0.01-0.02 $\AA$ shorter when BS3 is employed. The last basis set system tested, the polarised triple zeta valence def2-TZVP (BS5), leads to the largest number of basis functions among the tested basis set systems. Surprisingly, the PBEO/BS5 optimised structures are not as good as expected, an outcome probably related to the absence of pseudopotentials in the description of the Mo and $\mathrm{S}$ centres.

All in all, DFT calculations using the PBEO functional and its dispersion corrected D3(0) and $\mathrm{D} 3(\mathrm{BJ})$ versions in combination with the BS1 basis set constitutes the most accurate level of theory for optimisation purposes, leading to MUE values in the same range as the uncertainties of the X-ray diffraction determined interatomic distances.

\section{Activation free energies}

In spite of the fact that activation enthalpies and entropies have been obtained for the reaction of $[\mathbf{1}]^{+}$with dmad and adc in acetonitrile solution, herein we have focussed on the computation of the resulting Gibbs free energies in Table 4. This choice is based on the fact that the continuum solvation models employed herein, PCM and SMD, are constructed to calculate Gibbs free energies of solvation and do not provide direct information about separate enthalpies and entropies of solvation. ${ }^{1}$ A wide range of density functionals have been tested and the results are included in Table S5. This allows comparing several common variables when trying to compute free energy barriers. For instance, regardless of how solvent effects are accounted for, the values obtained at levels of theory that do not include dispersion effects (entries 2, 5, 8, 11 and 14, Table S5) clearly overestimate the barriers for the forward reactions R1 and R3, whereas they underestimate the backward reaction $\mathrm{R} 2$, these discrepancies being in the range of 5-10 $\mathrm{kcal} / \mathrm{mol}$. As expected, inclusion of dispersion effects on the computation of the free energy barriers leads to significant decreases in the barriers of R1 and R3, in agreement with the fact that such processes involve a change in molecularity from 2 to 1 . On the contrary, the effect is much smaller for the backward reaction in $\mathrm{R} 2$, where there is no change in molecularity.

Although the extent of the decrease on the $\Delta G^{\#}$ values for R1 and R3 differs between the D3(0) and $\mathrm{D} 3(\mathrm{BJ})$ corrections, with the latter being slightly more pronounced, such corrections are found to be generally too large, leading to an underestimation of the barriers. This is clearly exemplified when comparing the barrier for reaction R1 (experimental $\Delta G^{\#}$ value of 20.8 $\mathrm{kcal} / \mathrm{mol}$, entry 1) with the computed values for the BP86 functional without and with added dispersion corrections (entries 2-4, Table S5). Focusing on the PCM results, these calculations overestimate the barrier by $5.3 \mathrm{kcal} / \mathrm{mol}$ when no dispersion effects are considered, whereas it becomes underestimated by 11.1 and 14.9 $\mathrm{kcal} / \mathrm{mol}$ after inclusion of D3(0) and D3(BJ) corrections, respectively. The B3LYP results (entry 11, Table S5) are worth highlighting at this point, as they are the worst among the selected methodologies, overestimating the barriers for R1 and R3 by more than 15 $\mathrm{kcal} / \mathrm{mol}$.

As previously shown for the BP86 functional, the best performer in terms of computed geometries (PBE0) also overestimates the barriers for R1 and R3 (entry 14, Table S5), and underestimates them when dispersion corrections are included (entries 15 and 16, Table S5). The obtained MUE values are however much better in this latter case, in part because the barrier for R2 is well accounted for in the three cases. The family of M06 
functionals represent a nice opportunity to analyse how the \%HF exchange affects the computed energies. These functionals include dispersion effects in some extent, and therefore the D3(0) corrections are smaller than for other functionals and lead to minor changes in the barriers. Focussing first on the non-D3(0) corrected values in entries 17, 19, 21 and 23 (Table S5), a clear correlation is easily spotted for the backward reaction R2, whose barrier linearly increases with the \%HF exchange $\left(R^{2}=\right.$ 0.98 , see Figure S3) both for PCM and SMD solvent-corrected values). Among them, the data shows that the M06-2X variant is the best performer, being also worth noting that the addition of $\mathrm{D} 3(0)$ correction improves the results up to MUE values of 1.3 and 2.5 $\mathrm{kcal} / \mathrm{mol}$ for the PCM and SMD solvent corrected data, respectively (entry 22, Table S5). In relation to the \%HF exchange subject, it is observed that the range-separated hybrids M11 and WB97XD do not lead to significant improvements (entries 26 and 27, Table S5). The non-dispersion-corrected M11 functional generally overestimates barriers whereas the dispersion corrected WB97XD underestimates them.

In order to test whether it is more appropriate to include dispersion corrections during the optimisation procedure or afterwards as a single-point correction, optimisations were initially performed employing the D3(BJ) corrected versions of BP86, B3LYP, and PBEO functionals (entries 28-30, Table S5). As expected, comparison of the resulting free energy barriers with those in entries 4,13 , and 16 (Table S5) shows that this variable has relatively little effect on the outcome (differences smaller than $1 \mathrm{kcal} / \mathrm{mol}$ ). For the sake of completeness, optimisations were also performed with the PBEOD3(0) functional (entry 31, Table S5), which again led to small differences with respect to the results obtained by adding the $\mathrm{D} 3(0)$ correction to the PBEO energies (entry 15, Table S5).

The way in which solvent effects are taken into account in the calculations also represent an important ingredient of the computational methodology. Herein we have only compared the well-known PCM and SMD approaches. In general, the data obtained with the latter model leads to a small improvement on the free energy barriers, although the effect depends on the specific level of theory. All in all, the data in Table S5 indicates that M062XD3(0)(BS2,PCM)/M06-2X(BS1) and PBEOD3(0)(BS2,SMD)/PBEOD3(0)(BS1)

approaches, closely followed by PBE0D3(BJ)(BS2,SMD)/PBEO(BS1) and PBEOD3(BJ)(BS2,SMD)/PBEOD3(BJ)(BS1) are the most accurate among the tested ones. The dependence of the computed activation free energy barriers on the employed basis set system was analysed subsequently based on the PBEO/BS1 data (Table 6, entries 32-37). From the results in Table $S 5$ it has already been shown that the $\mathrm{D} 3(0)$ dispersion correction leads to lower MUE values than the D3(BJ) correction. The PBE0/BS1 structures have been used to confirm that this trend is independent of the employed basis set system, as clearly shown by the MUE values for entry $35>32,36$ $>33$, and $37>34$ in Table 6. Thus, focusing on the D3(0) corrected energies, the results indicate that indeed when BS1 (entry 32) is substituted by BS2 (entry 15) there is an average MUE improvement of ca. $3 \mathrm{kcal} / \mathrm{mol}$. The BS4 basis set system (entry 33 ) generates MUE values between those of BS2 and BS1, and therefore its use is not justified. On the contrary, despite its relatively poor performance for optimisation purposes, BS5 (entry 34 ) is found to be the best performer in terms of free energy barriers, in fact even better than BS2, reaching a MUE value of only $0.9 \mathrm{kcal} / \mathrm{mol}$ when combined with the SMD solvent model. Based on these findings, a similar basis set analysis was carried out on the PBEOD3(0)(BS1) optimised geometries (entries 38-40), which again showed that BS5 gives the best performance among the basis set systems tested for computing electronic energies. All in all, the results in this section indicate that the PBEOD3(0)(BS5,SMD)/PBE0D3(0)(BS1) level of theory allows achieving chemical accuracy $( \pm 1$ $\mathrm{kcal} / \mathrm{mol})$, as it results in an MUE value of 0.7 
errors.

\begin{tabular}{|c|c|c|c|c|c|c|c|c|c|}
\hline & & \multirow{2}{*}{\multicolumn{4}{|c|}{ Solvent $=$ PCM }} & \multirow{2}{*}{\multicolumn{4}{|c|}{ Solvent $=$ SMD }} \\
\hline \multirow[b]{2}{*}{$\begin{array}{l}\text { Entr } \\
\mathrm{y}\end{array}$} & \multirow[b]{2}{*}{ Level of theory } & & & & & & & & \\
\hline & & R1 & $\mathbf{R 2}$ & R3 & MUE & R1 & $\mathbf{R 2}$ & R3 & MUE \\
\hline 1 & Experimental & 20.8 & 23.3 & 17.7 & & 20.8 & 23.3 & 17.7 & \\
\hline 15 & PBE0D3(0)(BS2,Solvent)/PBE0(BS1) & 17.5 & 21.7 & 13.6 & 3.0 & 19.4 & 21.6 & 15.3 & 1.8 \\
\hline 32 & PBE0D3(0)(BS1,Solvent)/PBE0(BS1) & 13.6 & 22.6 & 7.5 & 6.0 & 15.7 & 22.6 & 9.2 & 4.8 \\
\hline 33 & PBE0D3(0)(BS4,Solvent)/PBE0(BS1) & 16.0 & 23.7 & 8.4 & 4.8 & 18.0 & 23.6 & 10.2 & 3.5 \\
\hline 34 & PBE0D3(0)(BS5,Solvent)/PBE0(BS1) & 18.0 & 23.1 & 14.4 & 2.1 & 19.9 & 23.0 & 16.2 & 0.9 \\
\hline 30 & PBE0D3(BJ)(BS2,Solvent)/PBE0(BS1) & 15.7 & 23.1 & 12.0 & 3.7 & 17.6 & 23.0 & 13.8 & 2.5 \\
\hline 35 & PBE0D3(BJ)(BS1, Solvent)/PBE0(BS1) & 11.8 & 24.0 & 5.9 & 7.2 & 13.9 & 23.9 & 7.6 & 5.9 \\
\hline 36 & PBE0D3(BJ)(BS4,Solvent)/PBE0(BS1) & 14.3 & 25.0 & 6.9 & 6.3 & 16.2 & 25.0 & 8.6 & 5.1 \\
\hline 37 & PBE0D3(BJ)(BS5,Solvent)/PBE0(BS1) & 16.2 & 24.4 & 12.9 & 3.5 & 18.2 & 24.3 & 14.6 & 2.2 \\
\hline 31 & PBE0D3(0)(BS2,Solvent)/PBE0D3(0)(BS1) & 17.8 & 21.8 & 13.6 & 2.9 & 19.9 & 21.8 & 15.3 & 1.6 \\
\hline 38 & PBE0D3(0)(BS1,Solvent)/PBE0D3(0)(BS1) & 13.8 & 22.9 & 7.3 & 5.9 & 16.2 & 23.0 & 8.7 & 4.6 \\
\hline 39 & PBE0D3(0)(BS4,Solvent)/PBE0D3(0)(BS1) & 16.3 & 24.0 & 8.3 & 4.9 & 18.5 & 24.0 & 9.8 & 3.6 \\
\hline 40 & PBE0D3(0)(BS5,Solvent)/PBE0D3(0)(BS1) & 18.3 & 23.4 & 14.4 & 2.0 & 20.5 & 23.4 & 15.9 & 0.7 \\
\hline
\end{tabular}

\section{Activation volumes}

The changes on the volumetric properties of a system along a reaction coordinate lead to the concept of volume profile. Similarly to energy profiles, within a volume profile it is possible to identify activation $\left(\Delta V^{\#}\right)$ and reaction volumes $(\Delta V) .{ }^{57}$ These magnitudes can be experimentally obtained by studying the effect of the pressure on the equilibrium and reaction constants of the process, as shown in Eq. 2 and 3. Moreover, within the framework of the transition state theory $\Delta V^{\#}$ and $\Delta V_{R}$ are related to the partial molar volumes of the products, transition state and reactants according to Eq. 4 and 5 . Interestingly, such correlations have been exploited by computational chemists to obtain theoretical volume profiles, which can be used to predict the structure of transition states by comparing them with experimentally determined activation volumes. ${ }^{58-59}$

$$
\begin{aligned}
& \Delta V=-R T\left(\frac{\delta \ln K}{\delta P}\right)_{T} \\
& \Delta V^{\#}=-R T\left(\frac{\delta \ln k}{\delta P}\right)_{T} \\
& \Delta V^{\#}=V^{\#}-V_{R} \\
& \Delta V=V_{P}-V_{R}
\end{aligned}
$$

For the concerted [3+2] cycloaddition processes studied herein, it provides important information in support of the proposed mechanism. Thus, the empirical negative activation volumes obtained for the forward reactions are a clear indication of the contraction associated with the TS formation from the separated species, whereas the positive value obtained for the reverse process highlights the expansion undergone by the dithiolene adducts in order dissociate the alkyne moiety at the TS structure. 
Interestingly, a specific computational method has been developed recently to study the effect of extreme pressure on reactions taking place in solution (XP-PCM), ${ }^{60-61}$ ultimately allowing to compute $\Delta V_{\text {reac }}$ and $\Delta V^{\#}$ values. ${ }^{62}$ Unfortunately, the range of pressures required to obtain $\Delta V^{\#}$ values via XP-PCM calculations goes from 0 to 8$10 \mathrm{GPa}$ (1 GPa= 104 bar), i.e. two orders of magnitude larger than the highest pressures typically employed in the experiments (0-103 bar). Herein we have instead computed $\Delta V^{\dagger}$ values based on the differences between the VdW volumes of transition state structures and reactants, a methodology successfully used for instance to study water exchange processes at transition metal complexes. ${ }^{63-64}$

The results so obtained, included in Table S6, are evidently very dependent on the way in which atomic radii are computed. By default, Gaussian09 uses different atomic radii models for the PCM and SMD approaches. Thus, while the scaled ( $f=1.1$ ) UFF is associated with PCM, an unscaled intrinsic atomic Coulomb radii is employed for SMD. The agreement between experimental and computed activation volumes in Table S6 is quite good in both cases. It is easily seen that the unscaled intrinsic atomic Coulomb radii leads to better results than the scaled UFF radii regardless of the employed DFT functional, with average MUE values of ca. 2 and $4 \mathrm{~cm}^{3} \mathrm{~mol}^{-1}$, respectively. Again, the errors are larger for R1 and R3 than for R2, an effect likely to be associated with the change in molecularity of the former reactions. As noted above, the computed $\Delta V^{\#}$ values show little dependence with the DFT functional at which the optimisations were carried out. This is not surprising given that, although the employed density functional has obviously an impact on the resulting structures (see above), those geometrical differences are not significant enough to provoke major changes in the resulting molecular VdW surfaces. All in all, the computations in this section show that the SMD solvation model predicts $\Delta V^{\#}$ values that in average deviate by ca. $2 \mathrm{~cm}^{3} \mathrm{~mol}^{-1}$ with respect to the empirical ones, a value in fact very similar to the uncertainty of these empirical measurements.

Performance of the optimised procedure when modelling single temperature kinetic data

As the kinetics of reactions are often measured at a single temperature (typically 25ㅇ) , we decided to test the performance of the optimised PBEOD3(0)(BS5, SMD)/PBEOD3(0)(BS1) protocol to model activation free energies obtained in this way. For this purpose, not only the reactions of [1] $]^{+}$ with adc and PrA (see Table 3) were tested, but also previously reported data for the related $\left[\mathrm{Mo}_{3} \mathrm{~S}_{4}(\mathrm{acac})_{3}(\mathrm{py})_{3}\right]^{+} \quad$ cluster $($ acac = acetylacetonate; py = pyridine), for which differences between experimental and computed free energies of up to $5 \mathrm{kcal} / \mathrm{mol}$ have been noted. ${ }^{14}$ Notably, from the reactions of [1] ${ }^{+}$a MUE value of $1.0 \mathrm{kcal} / \mathrm{mol}$ is obtained from the data in Table 7, thus indicating that the accuracy of the protocol remains relatively similar when tested on these alkynes. Furthermore, a similar MUE value results for the reactions of $\left[\mathrm{Mo}_{3} \mathrm{~S}_{4}(\mathrm{acac})_{3}(\mathrm{py})_{3}\right]^{+}$with alkynes, which therefore indicates that the protocol is also valid to study the reactivity of other clusters with a $\left[\mathrm{Mo}_{3}\left(\mu_{3}-\mathrm{S}\right)(\mu-S)_{3}\right]^{4+}$ core.

\begin{tabular}{|c|c|c|c|c|c|}
\hline & & & & & \\
\hline & & For & ard & Rev & rse \\
\hline & & Exp. & DFT & Exp. & DFT \\
\hline$[1]^{+}$ & adc & 17.7 & 15.7 & & \\
\hline & dmad & 21.0 & 22.0 & 24.3 & 24.3 \\
\hline$\left[\mathrm{Mo}_{3} \mathrm{~S}_{4}(\mathrm{acac})_{3}(\mathrm{py})_{3}\right]^{+}$ & adc & 16.2 & 14.4 & & \\
\hline & dmad & 15.4 & 15.5 & & \\
\hline & btd & 20.3 & 21.8 & & \\
\hline & PrA & 20.8 & 21.3 & 23.0 & 22.1 \\
\hline & $\mathrm{PhA}$ & 20.8 & 20.0 & 22.3 & 20.9 \\
\hline & FPhA & 20.3 & 20.6 & & \\
\hline & ${ }^{\mathrm{CF} 3} \mathrm{PhA}$ & 21.4 & 20.6 & & \\
\hline
\end{tabular}

\section{Conclusions}


Free energies $\left(\Delta G^{\#}\right)$ and volumes $\left(\Delta V^{\#}\right)$ of activation for the $[3+2]$ cycloaddition reaction between the $[\mathbf{1}]^{+}$cluster and two alkynes, dmad and $\mathrm{PhA}$, have been determined based on a kinetic study at different temperatures and pressures. These data, together with the already reported interatomic distances for the $\mathrm{X}$-ray diffraction structures of $[\mathbf{1}]\left(\mathrm{BF}_{4}\right)$ and [1dmad $]_{2}\left(\mathrm{Mo}_{6} \mathrm{Cl}_{14}\right)$ cluster salts have been subsequently employed to identify the most accurate computational protocol among those commonly employed for this purpose. To do so, different functionals and basis set systems have been studied and other important factors such as the inclusion of dispersion and solvent effects have also been taken into account. DFT calculations using the PBEO functional and its dispersion corrected PBEOD3(0) version are the most accurate among the tested functionals, with similar performances in terms of interatomic distances, but the dispersion correction (either as single-point or included self-consistently in the optimisation procedure) has proven to be necessary in order to obtain accurate energetic data. Interestingly, little functional dependence was observed on the computation of activation volumes, a magnitude that is much more dependent on the way in which van der Waals surfaces are constructed. In general, the SMD solvation model was found to give better results than the PCM approach both in terms of $\Delta G^{\#}$ and $\Delta V^{\#}$. Basis sets were also found to lead to significant changes both in terms of computed geometries and $\Delta G^{\#}$ values; BS1 and BS5 lead to the most accurate results when employed for geometry optimisation and subsequent single-point calculations, respectively. All in all, we concluded that the PBEOD3(0)(BS5,SMD)/PBEOD3(0)(BS1) protocol represents a good compromise between accuracy and computational cost, leading to a MUE value of only $0.7 \mathrm{kcal} / \mathrm{mol}$ for the $\Delta G^{\#}$ values computed for the reactions between $[\mathbf{1}]^{+}$ and the dmad and PhA alkynes. Thus, the computational protocol developed herein demonstrates that accurate estimations of the activation free energies and volumes can be obtained with an adequate choice of the computational method. Moreover, its predictive capability for the reaction of a different cluster indicates that it can be extended to other systems when a fine computational analysis of the structure and reactivity of $\left[\mathrm{Mo}_{3} \mathrm{~S}_{4}\right]^{4+}$ cuboidal clusters is required, and so we recommend its use.

\section{Acknowledgments}

Financial support from the Spanish Ministerio de Economía y Competitividad (CTQ201565707C2-1 and -2/FEDER and PGC2018-094417$\mathrm{B}-100)$ is acknowledged.

Keywords: DFT benchmarking $-3+2$

cycloaddition - Reaction mechanisms - Cluster

compounds - Alkynes

Additional Supporting Information may be found in the online version of this article.

\section{ORCID}

Rosa Llusar https://orcid.org/0000-0002-35397269

Manuel Martínez https://orcid.org/0000-00026289-4586

Manuel G. Basallote https://orcid.org/00000002-1802-8699

Andrés G. Algarra https://orcid.org/0000-00025062-2858

\section{References and Notes}

1. J. N. Harvey, F. Himo, F. Maseras, L. Perrin, ACS Catal., 2019, 9, 6803-6813.

2. S. Grimme, P. R. Schreiner, Angew. Chem., Int. Ed., 2018, 57, 4170-4176.

3. I. S. Edward, M. Kazuko, In Transition Metal Sulfur Chemistry, American Chemical Society, 1996.

4. A. L. Gushchin, Y. A. Laricheva, M. N. Sokolov, R. Llusar, Russ. Chem. Rev., 2018, 87, 670-706.

5. A. G. Algarra, M. J. Fernández-Trujillo, M. G. Basallote, Chem. Eur. J., 2012, 18, 5036-5046. 
6. J. Á. Pino-Chamorro, Y. A. Laricheva, E. Guillamón, M. J. Fernández-Trujillo, A. G. Algarra, A. L. Gushchin, P. A. Abramov, E. Bustelo, R. Llusar, M. N. Sokolov, M. G. Basallote, Inorg. Chem. 2016, 55, 9912-9922.

7. A. G. Algarra, M. G. Basallote, in Advances in Inorganic Chemistry, eds. R. van Eldik, C. D. Hubbard, Academic Press, 2017, vol. 70, pp. 311-342.

8. H. Wang, C. Li, P. Fang, Z. Zhang, J. Z. Zhang, Chem. Soc. Rev. 2018, 47, 61016127.

9. Z. Huang, W. Luo, L. Ma, M. Yu, X. Ren, M. He, S. Polen, K. Click, B. Garrett, J. Lu, K. Amine, C. Hadad, W. Chen, A. Asthagiri, Y. Wu, Angew. Chem. Int. Ed. 2015, 54, 15181-15185.

10. A. G. Algarra, E. Guillamón, J. Andrés, M. J. Fernández-Trujillo, E. Pedrajas, J. Á. Pino-Chamorro, R. Llusar, M. G. Basallote, ACS Catal., 2018, 8, 73467350.

11. E. Pedrajas, I. Sorribes, K. Junge, M. Beller, R. Llusar, ChemCatChem, 2015, 7, 2675-2681.

12. E. Pedrajas, I. Sorribes, A. L. Gushchin, Y. A. Laricheva, K. Junge, M. Beller, R. Llusar, ChemCatChem, 2017, 9, 11281134.

13. M. Vázquez, M. Martínez, Inorg. Chem., 2016, 55, 6731-6738.

14. R. A. Binstead, B. Jung, A. D. Zuberbühler, SPECFIT-32, Spectrum Software Associates, Chappel Hill, 2000.

15. M. Maeder, P. King, ReactLab, JPlus Consulting Pty Ltd., East Fremantle, Australia, 2009.

16. M. J. Frisch, G. W. Trucks, H. B. Schlegel, G. E. Scuseria, M. A. Robb, J. R. Cheeseman, G. Scalmani, V. Barone, B. Mennucci, G. A. Petersson, H. Nakatsuji, M. Caricato, X. Li, H. P. Hratchian, A. F. Izmaylov, J. Bloino, G. Zheng, J. L. Sonnenberg, M. Hada, M. Ehara, K. Toyota, R. Fukuda, J. Hasegawa, M. Ishida, T. Nakajima, Y. Honda, O. Kitao, H. Nakai, T. Vreven, J. A. Montgomery
Jr., J. E. Peralta, F. Ogliaro, M. Bearpark, J. J. Heyd, E. Brothers, K. N. Kudin, V. N. Staroverov, R. Kobayashi, J. Normand, K. Raghavachari, A. Rendell, J. C. Burant, S. S. lyengar, J. Tomasi, M. Cossi, N. Rega, J. M. Millam, M. Klene, J. E. Knox, J. B. Cross, V. Bakken, C. Adamo, J. Jaramillo, R. Gomperts, R. E. Stratmann, O. Yazyev, A. J. Austin, R. Cammi, C. Pomelli, J. W. Ochterski, R. L. Martin, K. Morokuma, V. G. Zakrzewski, G. A. Voth, P. Salvador, J. J. Dannenberg, S. Dapprich, A. D. Daniels, O. Farkas, J. B. Foresman, J. V. Ortiz, J. Cioslowski, D. J. Fox, Gaussian 09, Revision D.01, Gaussian, Inc., Wallingford CT 2009.

17. C. Gonzalez, H. B. Schlegel, J. Phys. Chem., 1990, 94, 5523-5527.

18. C. Gonzalez, H. B. Schlegel, J. Phys. Chem., 1991, 95, 5853-5860.

19. D. Andrae, U. Haussermann, M. Dolg, H. Stoll, H. Preuss, Theor. Chim. Acta, 1990, 77, 123-141.

20. A. Hollwarth, M. Bohme, S. Dapprich, A. W. Ehlers, A. Gobbi, V. Jonas, K. F. Kohler, R. Stegmann, A. Veldkamp, G. Frenking, Chem. Phys. Lett., 1993, 208, 237-240.

21. J.-L. Li, R. A. Mata, U. Ryde, J. Chem. Theory Comput., 2013, 9, 1799-1807.

22. L. $\mathrm{Hu}, \mathrm{H}$. Chen, J. Chem. Theory Comput., 2015, 11, 4601-4614.

23. F. Weigend, R. Ahlrichs, Phys. Chem. Chem. Phys., 2005, 7, 3297-3305.

24. K. A. Peterson, D. Figgen, M. Dolg, H. Stoll, J. Chem. Phys., 2007, 126, 124101.

25. A. K. Wilson, D. E. Woon, K. A. Peterson, T. H. Dunning, J. Chem. Phys., 1999, 110, 7667-7676.

26. T. H. Dunning, J. Chem. Phys., 1989, 90, 1007-1023.

27. J. P. Perdew, K. Schmidt, AIP Conference Proceedings, 2001, 577, 1-20.

28. J. Tomasi, B. Mennucci, R. Cammi, Chem. Rev., 2005, 105, 2999-3093.

29. A. V. Marenich, C. J. Cramer, D. G. Truhlar, J. Phys. Chem. B, 2009, 113, 6378-6396. 
30. J. H. Jensen, Phys. Chem. Chem. Phys., 2015, 17, 12441-12451.

31. A. Fernández-Ramos, B. A. Ellingson, R. Meana-Pañeda, J. M. C. Marques, D. G. Truhlar, Theo. Chem. Acc., 2007, 118, 813-826.

32. M. K. Gilson, K. K. Irikura, J. Phys. Chem. $B, 2010,114,16304-16317$.

33. A. D. Becke, Phys. Rev. A, 1988, 38, 3098.

34. J. P. Perdew, Phys. Rev. B, 1986, 33, $8822-8824$

35. S. Grimme, S. Ehrlich, L. Goerigk, J. Comput. Chem., 2011, 32, 1456-1465.

36. S. Grimme, J. Antony, S. Ehrlich, H. Krieg, J. Chem. Phys., 2010, 132, 154104.

37. S. Grimme, J. Comput. Chem., 2006, 27, 1787-1799.

38. J. Tao, J. P. Perdew, V. N. Staroverov, G. E. Scuseria, Phys. Rev. Lett., 2003, 91, 146401.

39. Y. Zhao, D. G. Truhlar, J. Chem. Phys., 2006, 125, 194101.

40. J. P. Perdew, J. Tao, V. N. Staroverov, G. E. Scuseria, J. Chem. Phys., 2004, 120, 6898-6911.

41. J. P. Perdew, S. Kurth, A. Zupan, P. Blaha, Phys. Rev. Lett., 1999, 82, 25442547.

42. A. D. Becke, J. Chem. Phys., 1993, 98, 5648-5652.

43. J. P. Perdew, M. Ernzerhof, K. Burke, J. Chem. Phys., 1996, 105, 9982-9985.

44. C. Adamo, V. Barone, J. Chem. Phys., 1999, 110, 6158-6170.

45. Y. Zhao, D. G. Truhlar, Theo. Chem. Acc., 2008, 120, 215-241.

46. Y. Zhao, D. G. Truhlar, J. Phys. Chem. A, 2006, 110, 13126-13130.

47. R. Peverati, D. G. Truhlar, J. Phys. Chem. Lett., 2011, 2, 2810-2817.

48. J.-D. Chai, M. Head-Gordon, Phys. Chem. Chem. Phys., 2008, 10, 66156620.

49. J.-D. Chai, M. Head-Gordon, J. Chem. Phys., 2008, 128, 084106.
50. M. Álvarez-Moreno, C. de Graaf, N. López, F. Maseras, J. M. Poblet, C. Bo, J. Chem. Inf. Model., 2015, 55, 95-103.

51. Y. Minenkov, Å. Singstad, G. Occhipinti, V. R. Jensen, Dalton Trans., 2012, 41, 5526-5541.

52. R. Kang, H. Chen, S. Shaik, J. Yao, J. Chem. Theory Comput., 2011, 7, 40024011.

53. M. P. Waller, H. Braun, N. Hojdis, M. Bühl, J. Chem. Theory Comput., 2007, 3, 2234-2242.

54. T. Sperger, I. A. Sanhueza, I. Kalvet, F. Schoenebeck, Chem. Rev., 2015, 115, 9532-9586.

55. S. Zhao, Z.-H. Li, W.-N. Wang, Z.-P. Liu, K.-N. Fan, Y. Xie, H. F. Schaefer, J. Chem. Phys., 2006, 124, 184102.

56. M. Bühl, C. Reimann, D. A. Pantazis, T. Bredow, F. Neese, J. Chem. Theory Comput., 2008, 4, 1449-1459.

57. P. Muller, Pure Appl. Chem., 1994, 66, 1077-1184.

58. H. Wiebe, M. Louwerse, N. Weinberg, J. Chem. Phys., 2017, 146, 104104.

59. J. Spooner, H. Wiebe, M. Louwerse, B. Reader, N. Weinberg, Can. J. Chem. 2017, 96, 178-189.

60. R. Cammi, in Frontiers of Quantum Chemistry, Springer, Singapore, 2018, pp. 273-288.

61. R. Cammi, C. Cappelli, B. Mennucci, J. Tomasi, J. Chem. Phys., 2012, 137, 154112.

62. B. Chen, R. Hoffmann, R. Cammi, Angew. Chem., Int. Ed., 2017, 56, 11126-11142.

63. M. Regueiro-Figueroa, D. EstebanGómez, R. Pujales-Paradela, L. CanedaMartínez, A. de Blas, C. Platas-Iglesias, Int. J. Quantum Chem., 2016, 116, 13881396.

64. C. D. Hubbard, R. van Eldik, Inorg. Chim. Acta, 2010, 363, 2357-2374. 


\section{GRAPHICAL ABSTRACT}

AUTHOR NAMES: Elena Pedrajas, José A. Pino-Chamorro, Montserrat Ferrer, M. Jesús Fernández-Trujillo, Rosa Llusar, Manuel Martínez, Manuel G. Basallote, Andrés G. Algarra

TITLE: Benchmarking of DFT methods using experimental free energies and volumes of activation for the cycloaddition of alkynes to cuboidal $\mathrm{Mo}_{3} \mathrm{~S}_{4}$ clusters

TEXT: Despite the pressure dependence of the rate of reactions allows obtaining its activation volume, this magnitude is rarely studied from a computational viewpoint. Along with their free energy barriers, herein the activation volume for the reaction of $\mathrm{Mo}_{3} \mathrm{~S}_{4}$ clusters with alkynes is determined and subsequently used to obtain a computational protocol featuring average errors of $0.7 \mathrm{kcal} \mathrm{mol}^{-1}$ and 2 $\mathrm{cm}^{3} \mathrm{~mol}^{-1}$, respectively.

GRAPHICAL ABSTRACT FIGURE:

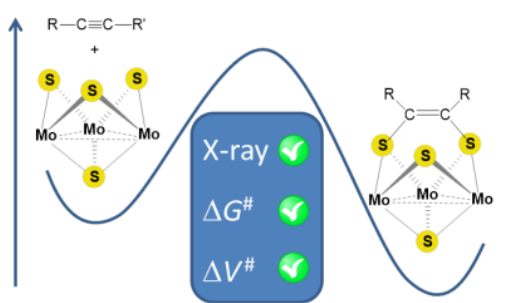

